AIDS. 2017 February 20; 31(4): 523-531. doi:10.1097/QAD.0000000000001367.

\title{
Outcomes of cervical cancer among HIV-infected and uninfected women treated at the Brazilian National Institute of Cancer (2001-2013)
}

\author{
Mariana P. Ferreira ${ }^{1,{ }^{*}}$, Anna E. Coghill ${ }^{2,}$, Claudia B. Chaves ${ }^{3,4}$, Anke Bergmann $^{4,5}$, Luiz C. \\ Thuler $^{5}$, Esmeralda A. Soares ${ }^{1}$, Ruth M. Pfeiffer ${ }^{2}$, Eric A. Engels ${ }^{2, \dagger}$, and Marcelo A. \\ Soares ${ }^{1, \dagger}$ \\ ${ }^{1}$ Programa de Oncovirologia, Instituto Nacional de Câncer, Rio de Janeiro, RJ, Brazil \\ ${ }^{2}$ Division of Cancer Epidemiology and Genetics, National Cancer Institute, Bethesda, MD, USA \\ ${ }^{3}$ Seção de Ginecologia Oncológica, Instituto Nacional de Cancer, Rio de Janeiro, RJ, Brazil \\ ${ }^{4}$ Programa de Carcinogênese Molecular, Instituto Nacional de Cancer, Rio de Janeiro, RJ, Brazil \\ ${ }^{5}$ Programa de Pesquisa Clínica, Instituto Nacional de Cancer, Rio de Janeiro, RJ, Brazil
}

\begin{abstract}
Objective-We assessed mortality, treatment response, and relapse among HIV-infected and HIV-uninfected women with cervical cancer in Rio de Janeiro, Brazil.

Design-Cohort study of 87 HIV-infected and 336 HIV-uninfected women with cervical cancer.

Methods-Patients at the Brazilian National Institute of Cancer (2001-2013) were matched on age, calendar year of diagnosis, clinical stage, and tumor histology. Staging and treatment with surgery, radiotherapy, and/or chemotherapy followed international guidelines. We used a Markov model to assess responses to initial therapy, and Cox models for mortality and relapse after complete response.
\end{abstract}

\begin{abstract}
Results-Among 234 deaths, most were from cancer (82\% in HIV-infected vs. 93\% in HIVuninfected women); only $9 \%$ of HIV-infected women died from AIDS. HIV was not associated with mortality during initial follow-up but was associated more than 1-2 years after diagnosis (overall mortality: stage-adjusted hazard ratio [HR] 2.02, 95\%CI 1.27-3.22; cancer-specific mortality: 4.35, 1.86-10.2). Among 222 patients treated with radiotherapy, HIV-infected had similar response rates to initial cancer therapy as HIV-uninfected women (HR 0.98, 95\%CI 0.581.66). However, among women who were treated and had a complete response, HIV was associated with elevated risk of subsequent relapse (HR 3.60, 95\% CI 1.86-6.98, adjusted for clinical stage).
\end{abstract}

Corresponding authors: Eric A. Engels, Division of Cancer Epidemiology and Genetics, National Cancer Institute, 9609 Medical Center Drive, Room 6E226, Bethesda, MD 20892 USA, Telephone 1-240-276-7186, engelse @exchange.nih.gov. Marcelo A. Soares, Program of Oncovirology, Instituto Nacional de Câncer, Rua André Cavalcanti, 37 - 4o andar, Bairro de Fátima, 20231-050 Rio de Janeiro, RJ, Brazil, Telephone 55-21-3207-6591, masoares@inca.gov.br.

* These authors contributed equally to this work.

$\dagger$ These authors contributed equally to this work. 
Conclusion-Among women with cervical cancer, HIV infection was not associated with initial treatment response or early mortality, but relapse after attaining a complete response and late mortality were increased in those with HIV. These results point to a role for an intact immune system in control of residual tumor burden among treated cervical cancer patients.

\section{Keywords}

HIV infection; AIDS; cervical cancer; treatment response; relapse; survival

\section{Introduction}

Infection with human immunodeficiency virus (HIV) increases risk for some malignancies, including cervical cancer. ${ }^{1,2}$ Cervical cancer is caused by human papillomavirus (HPV), and HIV-infected women are more likely than uninfected women to develop cervical HPV infections, including those with multiple infecting HPV types (a condition associated with progression to cervical cancer); less likely to clear HPV; and more likely to progress through pre-neoplastic stages to develop cervical cancer. ${ }^{3,4}$ The association between HIV and cancer is partly attributable to the effects of long-term and progressive immunosuppression. ${ }^{5}$ Highly active antiretroviral therapy (HAART), available since 1996, has led to prolonged survival in HIV-infected people. ${ }^{6,7}$

Cervical cancer causes substantial morbidity and mortality among women in developing countries. ${ }^{8}$ It is the third most common cancer in Brazilian women, and as estimated by the Brazilian National Institute of Cancer (INCA), over 16,000 cases will be diagnosed in 2016. ${ }^{9}$ In Brazil, women generally are diagnosed with cervical cancer at a late stage, ${ }^{10,11}$ as many do not receive Pap smear screening due to lack of healthcare access. HPV vaccination of adolescent girls was introduced in Brazil in 2014, ${ }^{12}$ but it will be decades before vaccination impacts cervical cancer trends.

HIV-infected cancer patients experience elevated overall mortality compared with uninfected cancer patients. ${ }^{13-15}$ This excess is partly due to acquired immunodeficiency syndrome (AIDS), but an adverse effect specifically on cancer outcomes is biologically plausible if an intact immune system helps control cancer after treatment. In a large registry-based study in the United States, ${ }^{14} \mathrm{HIV}$ was associated with a non-significant $27 \%$ increase in cancerspecific mortality among women with cervical cancer. That study utilized data from cancer registries, so it could not fully assess cancer stage and treatment, and it lacked data on treatment response and relapse. In a study of cervical cancer patients in Botswana, most patients presented with advanced stage tumors. ${ }^{16}$ Even though HIV-infected and HIVuninfected women were equally likely to have a complete tumor response to initial treatment, HIV infection was associated with a doubling in overall mortality, and more than $97 \%$ of deaths were attributed to patients' cancer. $^{16}$

Given availability of HAART, many HIV-infected women with cervical cancer will not die from AIDS, so it is important to understand the impact of HIV on cancer outcomes. Since 1996, HIV treatment with HAART has been universally available in Brazil through the public system. In the present study, we assessed Brazilian women treated for cervical cancer at INCA during 2001-2013. HIV-infected and HIV-uninfected women were closely matched 
on clinical stage and cancer treatment, and both early and late cancer outcomes were evaluated.

\section{Methods}

\section{Patients and data collection}

INCA is the Brazilian national cancer center and sets guidelines for cancer prevention, treatment, and surveillance. Initial diagnostic evaluation of cervical cancer patients in the state of Rio de Janeiro, Brazil, is conducted in basic healthcare units. Upon biopsy confirmation, patients are directed to the Gynecological Oncology Service of INCA Cancer Hospital II. At the initial appointment, a complete history and physical examination are performed, and referring pathology specimens and reports are reviewed. Clinicians ascertain HIV infection through clinical history and, if needed, HIV serology testing.

Clinical staging of cervical cancer is determined at INCA according to International Federation of Gynecology and Obstetrics (FIGO) standards. ${ }^{17-19}$ Laboratory and imaging exams are obtained to complement clinical staging, and patients are treated according to FIGO guidelines. ${ }^{17-19}$ Women with local stage cancer are typically treated with surgery, or radiation plus chemotherapy. Regional or distant stage cancer is treated with radiation plus chemotherapy. All surgeries are performed at INCA. Chemotherapy and radiotherapy may be performed at INCA, depending on the decision of state medical authorities. Some women with advanced cancer decide, in concert with their physicians, to receive palliative care only.

All patients are followed in outpatient appointments at INCA after completion of therapy. Follow-up begins 1 month after surgery or 4 months after brachytherapy, and all patients are seen every 6 months during the first 2 years and then yearly. At these appointments, a gynecological exam is performed, a specimen is collected for vaginal cytology, and imaging is obtained to evaluate for disease recurrence. If there is suggestive evidence of recurrence or progression, suspect lesions are biopsied. After 5 years, patients without evidence of cancer relapse are discharged.

Most deaths in INCA patients are identified through routine hospital procedures. First, some patients die while under treatment within INCA. Second, some deaths are reported by families to INCA's social service department. Third, when patients miss scheduled appointments, staff contact the family to determine the reason. We ascertained additional deaths $(\mathrm{N}=23$, or $10 \%$ of all deaths) through INCA's cancer registry, which is periodically linked to the state of Rio de Janeiro's mortality records.

Of 8744 women treated for cervical cancer at INCA during 2001-2013, $5471(62.5 \%)$ had an HIV serology test result in the hospital database. We identified $82 \mathrm{HIV}$-infected patients with positive HIV serology results in this way. We also searched for women who had CD4 or HIV viral load tests performed at INCA, which identified 5 additional women whose HIV-infected status was confirmed by review of clinical charts. A limitation of our approach is that we missed women with HIV documented only by clinical history or a handwritten note in the clinic chart. However, the chart review insured that no women were incorrectly assigned as HIV-infected. 
For each HIV-infected cervical cancer patient, we identified up to $4 \mathrm{HIV}$-uninfected patients matched on histologic subtype of cancer (squamous cell carcinoma vs. adenocarcinoma), clinical stage, treatment, and age ( \pm 5 years). We also sought to match closely on calendar year of registration ( $94 \%$ were matched within \pm 3 years). We confirmed lack of HIV infection through chart review.

Using a structured form, we abstracted data from INCA clinical charts and the hospital database regarding demographic characteristics, clinical stage, and treatment. We recorded tobacco and alcohol use based on standardized data collected at INCA registration (information included current, previous, or no use, but not specific burden). We linked HIVinfected women to the Ministry of Health HIV database to obtain CD4 counts measured at outside laboratories covered by the Brazilian public health system.

The study was approved by the INCA Ethics Committee (\#637.891).

\section{Statistical analyses}

We assessed predictors of overall mortality and cancer-specific mortality using Cox models. The timescale was time since cervical cancer diagnosis. Women were followed from INCA registration (delayed entry was utilized to account for any lag between diagnosis and registration) until the earliest of death, loss to follow-up, or 5 years after INCA registration. We present unadjusted hazard ratios (HRs) for demographic and clinical characteristics. For the overall patient group, we assessed the associations of HIV with mortality adjusted for clinical stage. We also assessed models restricted to patients treated with surgery or radiotherapy, with follow-up beginning at initiation of that treatment. Because all women treated with surgery had local stage cancer (i.e., stage I), stage adjustment was not done. Regression models for patients treated with radiotherapy were adjusted for clinical stage (categorized as IA/IB1, IB2/II, III, IVA/IVB) and, as a time-dependent covariate, administration of brachytherapy. We detected a possible interaction of HIV and time since diagnosis (i.e., non-proportionality), so we used $\log (-\log )$ plots to identify separate early and late follow-up periods for analysis.

Additionally, we fit multistate Markov models to assess the impact of HIV on initial treatment response, limited to women treated with radiotherapy (with or without chemotherapy). This approach allowed us to consider multiple outcomes of interest, including those for which the exact onset was not known exactly (interval censoring). The models assessed transitions from the initial state "ill with cervical cancer" to one of several outcomes: progression, cancer-specific mortality, death from other causes, and complete response. For progression, follow-up started at the beginning of treatment and ended at the earliest of recurrence, death, 8 months after end of treatment, or loss to follow-up. For death outcomes and complete response, follow-up started at completion of treatment and ended at the earliest of death, 8 months after end of treatment, or loss to follow-up. Cox models included terms for HIV and clinical stage, and left truncation accounted for delayed entry. While death dates were known, we did not observe exact dates for recurrence and complete response, but only that they occurred before a particular visit or between two dates. 
Finally, we compared risk of relapse among women who responded completely to initial therapy. Follow-up started 8 months after end of therapy (a period consistent with evaluation for complete response), excluding women who died or progressed before this date. We considered relapse to occur if a patient had a relapse documented at INCA or died due to cervical cancer. Patients were followed until one of these events, loss to follow-up by INCA, or 5 years after the start of analysis (i.e., 68 months after completion of therapy), whichever occurred first. Kaplan-Meier curves were constructed estimating the proportion of relapsefree women, and Cox models were used to measure the association between HIV and relapse.

For Markov models, we used the msm package (R, 2.15.3) to accommodate intervalcensored observations. ${ }^{20}$ Other statistical analyses utilized SAS (version 9.3) or SPSS (version 23).

\section{Results}

We assessed 87 HIV-infected women and 336 HIV-uninfected women with cervical cancer. Among HIV-infected women, 43 (49\%) had a CD4 count measured prior to cancer treatment, and for those with data, the median CD4 count closest to treatment was 263 cells $/ \mathrm{mm}^{3}$ (interquartile range 137-368). Fifty-five HIV-infected women (63\%) received HAART at some point during care at INCA.

Patient characteristics are presented in Table 1. By design, the two groups were matched well on age, calendar year of diagnosis, clinical stage, treatment, and histologic subtype of cervical cancer. The median age at diagnosis was 42 years. Overall, $93 \%$ of cases were squamous cell carcinomas. One-third of women (33\%) presented with stage I cancer, 17\% with stage II cancer, $40 \%$ with stage III cancer, and $11 \%$ with stage IV cancer.

Patients were treated based on clinical stage with surgery (28\%), radiation alone (23\%), or radiation and chemotherapy (30\%), while 151 patients (36\%) received additional brachytherapy. Most women completed recommended treatment (70\% HIV-infected women, $76 \%$ HIV-uninfected women). Due to patient preference, $19 \%$ of women received no cancerdirected therapy. Twenty-one HIV-infected women (24\%) and 89 HIV-infected women (26\%) were censored due to loss to follow-up within 5 years of INCA registration.

Fifty-eight HIV-infected and 176 HIV-uninfected women died. Among HIV-infected women, overall mortality was 324 per 1000 person-years, with $82 \%$ of deaths due to cancer (cancer-specific mortality, 266 per 1000 person-years) and 9\% due to AIDS (AIDS-specific mortality, 29 per 1000 person-years). Among HIV-uninfected women, overall mortality was 209 per 1000 person-years, with 93\% of deaths from cancer (cancer-specific mortality, 194 per 1000 person-years). Two-hundred twenty-seven deaths (97\% of all deaths) occurred in the first 5 years after INCA registration and were included in mortality analyses; the remaining 7 deaths occurred subsequently and were not analyzed.

Predictors of overall and cancer-specific mortality are shown in Table 2. HIV infection was associated with significantly elevated overall mortality (HR 1.38, 95\%CI 1.02-1.87) and a non-significant elevation in cancer-specific mortality (HR 1.31, 95\%CI 0.94-1.82). As 
expected, advanced clinical stage was strongly associated with worse overall mortality and especially cancer-specific mortality. Squamous cell carcinoma was also associated with higher overall and cancer-specific mortality than adenocarcinoma. Higher BMI, greater education, and non-smoking status were each associated with reduced mortality in unadjusted analyses. Among HIV-infected women, availability of a CD4 count before cancer treatment was associated with lower mortality.

As shown in Table 3, the associations of HIV infection with mortality were attenuated and not significant when adjusted for clinical stage (overall mortality HR $1.29,95 \% \mathrm{CI} 0.95-$ 1.75 ; cancer-specific mortality HR $1.18,95 \% \mathrm{CI} 0.85-1.65)$. Other variables were not significantly associated with mortality when added to these models and did not appreciably affect the HRs for HIV (not shown).

Among patients treated with surgery (all of whom had stage I cancer), HIV was associated with substantially elevated overall mortality (HR 8.70, 95\%CI 1.59-47.5). This result was based on few deaths $(\mathrm{N}=6)$, so adjustment was not possible, and because there was only one cancer death among women treated with surgery, the association of HIV with cancer-specific mortality could not be assessed. Among patients treated with radiotherapy, HIV was not associated with significantly elevated overall or cancer-specific mortality in adjusted models (Table 3).

Associations of HIV with overall and cancer-specific mortality appeared to vary over time ( $\mathrm{p}=0.04$ and $\mathrm{p}=0.12$, respectively). Based on $\log (-\log$ ) plots, we divided follow-up into early and late periods, at 1 year for overall mortality and 2 years for cancer-specific mortality. HIV was not associated with either outcome in the early follow-up period (Table 3) but was associated in the late period with overall mortality (stage-adjusted HR 2.02, 95\%CI 1.27$3.22)$ and cancer-specific mortality $(4.35,1.86-10.2)$.

Table 4 presents results of the Markov model examining HIV and early treatment outcomes among 218 women treated with radiotherapy ( 4 women were missing treatment dates and could not be evaluated). HIV was not associated with progression or cancer-specific mortality, or with a decrease in complete response. There were too few non-cancer deaths to assess the association with HIV.

Among treated women who had a complete response, $36 \mathrm{HIV}$-infected women and 168 uninfected women were alive and relapse-free 8 months after completion of therapy. Subsequently, 14 HIV-infected women and 26 uninfected women in this group relapsed. As shown in Figure 1, HIV was associated with substantially increased risk of relapse (unadjusted HR 2.84, 95\%CI 1.48-5.45; adjusted for clinical stage: HR 3.60, 95\% CI 1.86$6.98)$.

\section{Discussion}

Brazil faces major public health challenges seen in developing nations, including a substantial burden of cervical cancer, but with resources that allow for centers of excellence in cancer care. Our study setting at INCA allowed us access to a large retrospective clinical cohort of HIV-infected and HIV-uninfected women with cervical cancer who received 
standard-of-care evaluation and therapy. Overall mortality appeared modestly higher among HIV-infected women, but differences in overall and cancer-specific mortality were much stronger in the period more than 1-2 years after diagnosis. Moreover, among women who had a complete response to initial cancer therapy, HIV infection was associated with substantially elevated risk of subsequent cancer relapse.

There are no population-based data on the prevalence of HIV infection among cervical cancer patients in Brazil. The estimated HIV prevalence in the Brazilian general population is $0.39 \%,{ }^{21}$ but there is no separate estimate for women. At INCA, we had the chance to evaluate the HIV prevalence among the almost 5500 women with cervical cancer for whom an HIV serology test was available. The rate of infection was $1.9 \%$, approximately 5 -fold higher than in the general population in the country.

Overall mortality rates in our HIV-infected patient population (324 per 1000 person-years) appeared similar to that recently reported in Botswana (approximately $30 \%$ of patients deceased one year after diagnosis), and the majority of deaths in the patients in both countries were from cervical cancer. ${ }^{16}$ Mortality was lower than observed in similar patients in Uganda (65\% of HIV-infected women deceased 1 year after diagnosis) but still higher than in HIV-infected cervical cancer patients in the US (138 per 1000 person-years). ${ }^{13,14} \mathrm{~A}$ difference in mortality between Brazilian and US cervical cancer patients is also present for HIV-uninfected women and reflects much higher mortality among Brazilian patients with late stage cervical cancer (Supplemental Table 1), although the reasons for the worse outcomes in this group are unclear.

In the present study, HIV infection was not associated with early cancer outcomes. There was only one early cancer-related death among women with localized cervical cancer treated with surgery. Among women treated with radiotherapy, HIV was not associated with increased progression or cancer-specific mortality, or with a decrease in complete response, although the confidence limits for the HRs were wide. Likewise, in Botswana HIV-infected and HIV-uninfected patients with cervical cancer were equally likely to have complete or nearly complete tumor responses. ${ }^{16}$ These results suggest that HIV infection does not negatively affect initial control of cervical cancer achieved with standard treatment approaches.

In striking contrast, however, we observed greatly elevated risk for adverse late outcomes among HIV-infected women. We assessed women documented to have an initial complete response following stage-appropriate surgery and radiation. Among this group, HIV was associated with an elevated risk of cancer relapse (HR 3.60, 95\%CI 1.86-6.98, adjusted for cancer stage). Similarly, in our analyses of follow-up more than 1-2 years after diagnosis, we observed 2-4-fold increases in overall and cancer-specific mortality with HIV. In Botswana, mortality among HIV-infected cervical cancer patients (most of which was due to cancer) appeared elevated even in women with CD4 counts above 500 cells $/ \mathrm{mm}^{3}$ but was greatly increased among patients with the lowest $\mathrm{CD} 4$ counts. ${ }^{16}$

Taken together, these results suggest that an intact immune system is important for long-term control of microscopic foci of cancer, which likely remain present in some patients who 
respond completely to initial therapy, and that this control is suboptimal in HIV-infected patients. At least two additional lines of evidence support this hypothesis. First, patients who have had an apparently complete response to cancer treatment, including those disease-free for a number of years, can experience unexpected relapse following immunosuppression administered for solid organ transplantation, ${ }^{22}$ which would imply that their immune system had been controlling cancer at a subclinical level. Second, recently introduced therapies that target immune checkpoint molecules on the surface of tumor cells and lymphocytes (e.g., CTLA-4, PD-1, PD-L1) are effective at activating an anti-tumor immune response and lead to improved long-term outcomes for patients with a wide range of cancers. ${ }^{23,24}$

An important limitation of our study is that there were too few women with documented CD4 counts for us to assess the relationship between level of immunosuppression and early or late cancer outcomes. Based on limited data, most HIV-infected women in our study appeared to present with low CD4 counts indicative of advanced HIV infection. Among HIV-infected women in our study, overall mortality was lower in those who had a CD4 count recorded, consistent with engagement into HIV care. However, AIDS was directly responsible for only a minority (9\%) of deaths in our HIV-infected patients. In Brazil, HIVinfected people are eligible for free HIV care through the public health system. HAART treatment access follows international standards, being recommended to HIV-infected individuals with CD4 counts below 350 cells/ $/ \mathrm{mm}^{3}$ since 1996 and to any HIV-infected person irrespective of CD4 counts or clinical status since 2014. Currently, over $62 \%$ of the people with known HIV infection in Brazil receive antiretroviral therapy. Although the HIVinfected women included in the present study would have been eligible for HIV treatment under the national policy, the use of antiretroviral medications was likely captured incompletely in the Ministry of Health HIV database (not shown) and INCA clinical charts.

A strength of our study is that HIV-infected and uninfected patients were closely matched on most characteristics, treated at a single institution, and received evaluation and cancer care following international guidelines. ${ }^{17-19} \mathrm{~A}$ limitation is the small number of outcomes, especially for analyses restricted according to clinical stage, treatment, or follow-up interval. Additionally, our search of laboratory records in the hospital electronic database likely missed some patients known to be HIV-infected at the time of presentation to INCA, since those women may not have been tested again for HIV. Nonetheless, we confirmed the HIV status of all of the women included in our study through review of their clinic charts. Finally, our study relied on a combination of data sources to ascertain deaths. The great majority of deaths were ascertained through standard clinical follow-up procedures at INCA, which allows us to be somewhat confident regarding the cause of death. A small number of deaths were identified only through linkage with the Rio de Janeiro cancer registry. It is possible that the cause of death was misattributed in those cases, or that additional deaths were missed (e.g., if women moved out of the state of Rio de Janeiro).

Cervical cancer presents a major disease burden in Brazil. ${ }^{9}$ Not surprisingly, we found that advanced stage at cancer diagnosis and lower BMI (probably reflective of some degree of cancer-related cachexia) were associated with high mortality. Although preventative screening is available through the public health system, women with cervical cancer often present without a history of screening and with advanced stage disease. ${ }^{10,11}$ This situation 
reflects difficulties accessing medical care among women who are poor, lack an education, or live in areas where it is difficult to reach clinical services. Brazil has recently implemented nationwide HPV vaccination for $9-13$ year-old girls, ${ }^{12}$ which will likely reduce cervical cancer incidence over time.

In conclusion, we demonstrate that HIV-infected patients have an elevated risk of relapse of cervical cancer after achieving a complete response to initial therapy. Additional research is needed to better understand the contribution of the immune system in preventing such relapses. Although these patients were treated at a center of excellence in cancer care, overall mortality was high, largely due to deaths from cancer. The additional contribution of AIDS to overall mortality, while relatively small in our patient group, highlights the need for cancer patients to utilize appropriate HAART regimens.

\section{Supplementary Material}

Refer to Web version on PubMed Central for supplementary material.

\section{Acknowledgments}

This study was supported by a grant under the US-Brazil Collaborative Research Program of the US National Institutes of Health and the Brazilian Ministries of Health and of Science and Technology (\#467874/2014-0). MPF was supported through an MSc fellowship by the Brazilian Ministry of Health. CBC, AB, LCT, EAS and MAS were supported by intramural resources of the Brazilian Ministry of Health. AEC, RMP, and EAE were supported by the Intramural Research Program of the US National Cancer Institute.

We are indebted to the heads of the routine services at INCA that provided information and allowed data collection for this study, especially the Gynecologic Oncology Hospital II, the Information Technology section (André Cordeiro and Marcos Ferreira), the Cancer Hospital II Registry (Fernando Paiva Filho), and the Department of STD/AIDS of the Brazilian Ministry of Health (Silvana Giozza). We would also like to thank the INCA DirectorGeneral and the Healthcare Coordinator for providing clearance for the study to be conducted. Suzana Aguiar provided additional help with the database construction, organization, and codification.

\section{References}

1. Engels EA, Biggar RJ, Hall HI, et al. Cancer risk in people infected with human immunodeficiency virus in the United States. Int J Cancer. 2008; 123:187-194. [PubMed: 18435450]

2. Grulich AE, van Leeuwen MT, Falster MO, et al. Incidence of cancers in people with HIV/AIDS compared with immunosuppressed transplant recipients: a meta-analysis. Lancet. 2007; 370:59-67. [PubMed: 17617273]

3. Chaturvedi AK, Madeleine MM, Biggar RJ, et al. Risk of human papillomavirus-associated cancers among persons with AIDS. J Natl Cancer Inst. 2009

4. Denny LA, Franceschi S, de Sanjose S, et al. Human papillomavirus, human immunodeficiency virus and immunosuppression. Vaccine. 2012; 30(Suppl 5):F168-74. [PubMed: 23199960]

5. Guiguet M, Boue F, Cadranel J, et al. Effect of immunodeficiency, HIV viral load, and antiretroviral therapy on the risk of individual malignancies (FHDH-ANRS CO4): a prospective cohort study. Lancet Oncol. 2009; 10:1152-9. [PubMed: 19818686]

6. Detels R, Tarwater P, Phair JP, et al. Effectiveness of potent antiretroviral therapies on the incidence of opportunistic infections before and after AIDS diagnosis. AIDS. 2001; 15:347-355. [PubMed: 11273215]

7. Palella FJ, Delaney KM, Moorman AC, et al. Declining morbidity and mortality among patients with advanced human immunodeficiency virus infection. N Engl J Med. 1998; 338:853-860. [PubMed: 9516219] 
8. Fitzmaurice C, Dicker D, Pain A, et al. The Global Burden of Cancer 2013. JAMA Oncol. 2015; 1:505-527. [PubMed: 26181261]

9. Estimativa. incidência de câncer no Brasil. Instituto Nacional de Câncer José Alencar Gomes da Silva; Rio de Janeiro: 2016. http://www.inca.gov.br/estimativa/2016/ [accessed May 26, 2016]

10. Thuler LC, de Aguiar SS, Bergmann A. Determinants of late stage diagnosis of cervical cancer in Brazil. Rev Bras Ginecol Obstet. 2014; 36:237-43. [PubMed: 25099462]

11. Lourenco AV, Fregnani CM, Silva PC, et al. Why are women with cervical cancer not being diagnosed in preinvasive phase? An analysis of risk factors using a hierarchical model. Int $\mathbf{J}$ Gynecol Cancer. 2012; 22:645-53. [PubMed: 22343973]

12. Baker ML, Figueroa-Downing D, Chiang ED, et al. Paving pathways: Brazil's implementation of a national human papillomavirus immunization campaign. Rev Panam Salud Publica. 2015; 38:1636. [PubMed: 26581058]

13. Coghill AE, Newcomb PA, Madeleine MM, et al. Contribution of HIV infection to mortality among cancer patients in Uganda. Aids. 2013; 27:2933-42. [PubMed: 23921614]

14. Coghill AE, Shiels MS, Suneja G, et al. Elevated Cancer-Specific Mortality Among HIV-Infected Patients in the United States. J Clin Oncol. 2015; 33:2376-83. [PubMed: 26077242]

15. Marcus JL, Chao C, Leyden WA, et al. Survival among HIV-infected and HIV-uninfected individuals with common non-AIDS-defining cancers. Cancer Epidemiol Biomarkers Prev. 2015; 24:1167-73. [PubMed: 25713023]

16. Dryden-Peterson S, Bvochora-Nsingo M, Suneja G, et al. HIV Infection and Survival Among Women With Cervical Cancer. J Clin Oncol. 2016

17. National Comprehensive Cancer Network. [Accessed April 23, 2016] Cervical cancer, version 1.2012. Available online at http://www.nccn.org

18. Chemoradiotherapy for Cervical Cancer Meta-analysis Collaboration: Reducing uncertainties about the effects of chemoradiotherapy for cervical cancer: a systematic review and meta-analysis of individual patient data from 18 randomized trials. J Clin Oncol. 2008; 26:5802-12. [PubMed: 19001332]

19. Trimble EL, Harlan LC, Gius D, et al. Patterns of care for women with cervical cancer in the United States. Cancer. 2008; 113:743-9. [PubMed: 18618500]

20. Jackson CH. Multi-state models for panel data: the msm package for R. J Stat Software. 2011; 38:1-28.

21. Boletim Epidemiológico HIV/AIDS 2015 Ano IV. Vol. 1. Ministério da Saúde - Secretaria de Vigilância em Saúde - Departamento de DST, Aids e Hepatites Virais; Brasília: http:// www.aids.gov.br/sites/default/files/anexos/publicacao/2015/58534/ boletim_aids_11_2015_web_pdf_19105.pdf [accessed November 22, 2016]

22. Arron ST, Raymond AK, Yanik EL, et al. Melanoma Outcomes in Transplant Recipients With Pretransplant Melanoma. Dermatol Surg. 2016; 42:157-66. [PubMed: 26818209]

23. Brahmer JR, Tykodi SS, Chow LQ, et al. Safety and activity of anti-PD-L1 antibody in patients with advanced cancer. N Engl J Med. 2012; 366:2455-65. [PubMed: 22658128]

24. Hodi FS, O'Day SJ, McDermott DF, et al. Improved survival with ipilimumab in patients with metastatic melanoma. N Engl J Med. 2010; 363:711-23. [PubMed: 20525992] 


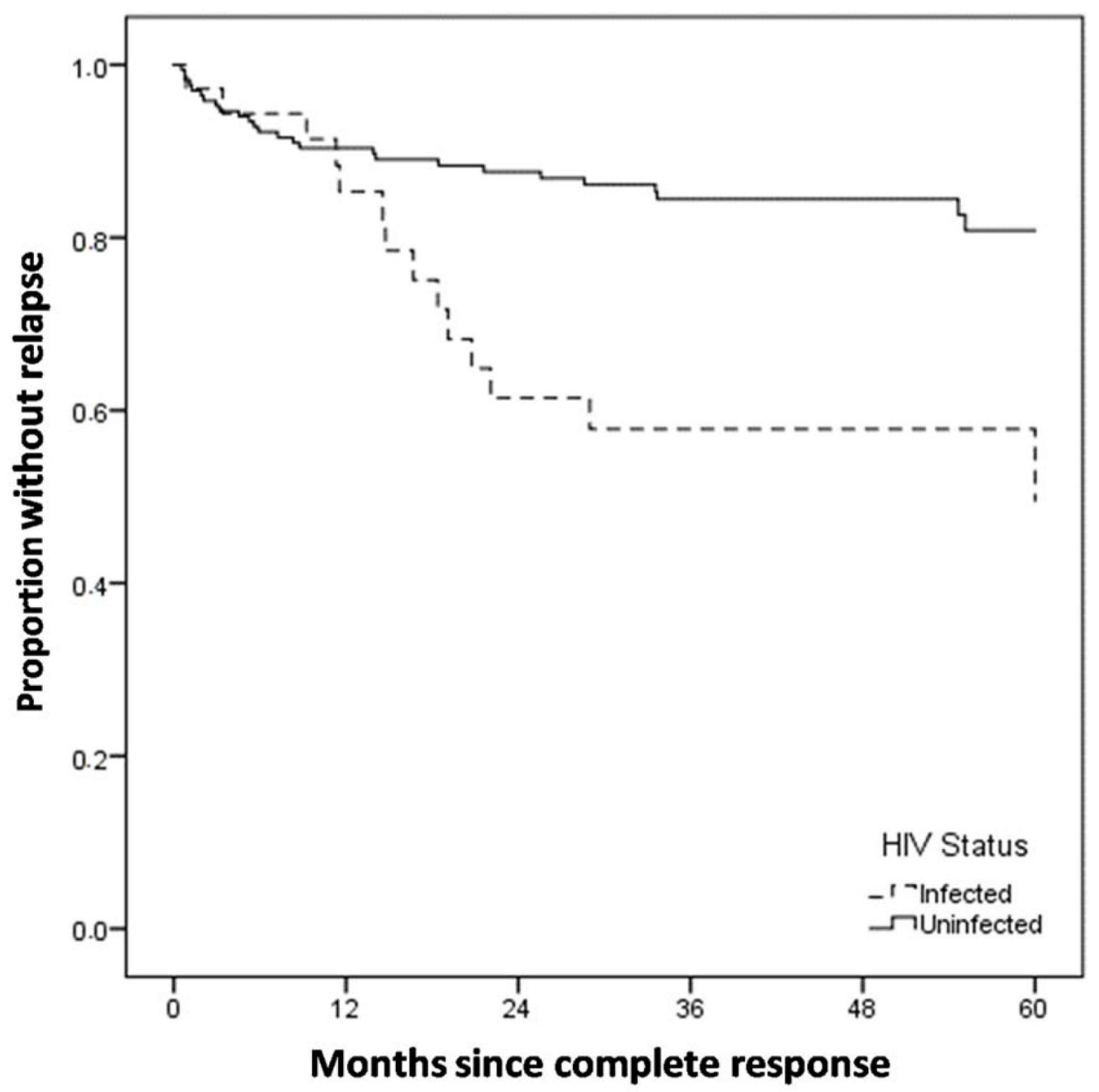

Figure 1.

Kaplan-Meier analysis of relapse among HIV-infected and uninfected patients with complete initial response to cancer therapy 


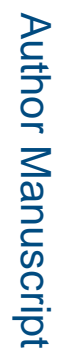

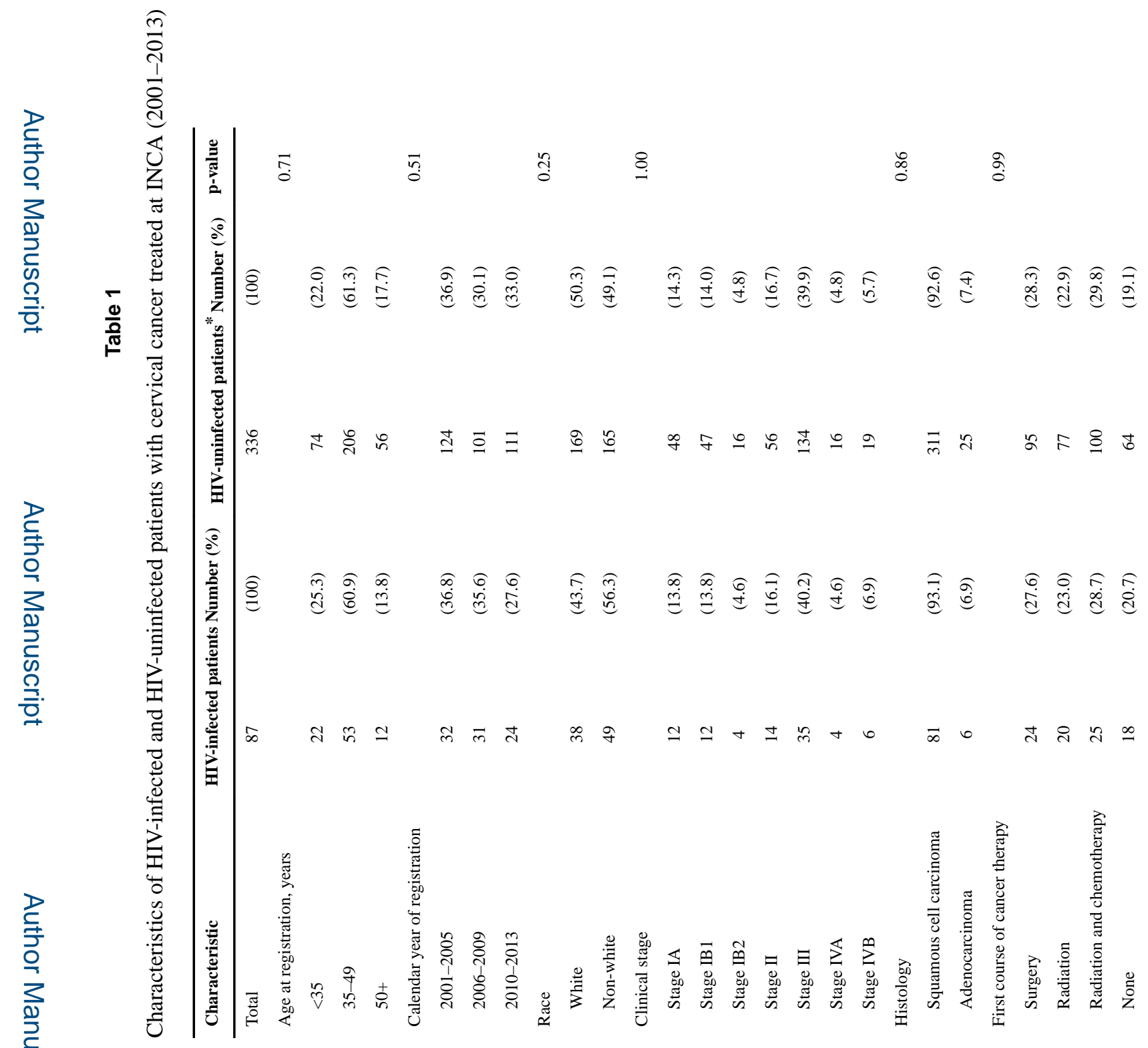


Ferreira et al.

Page 13

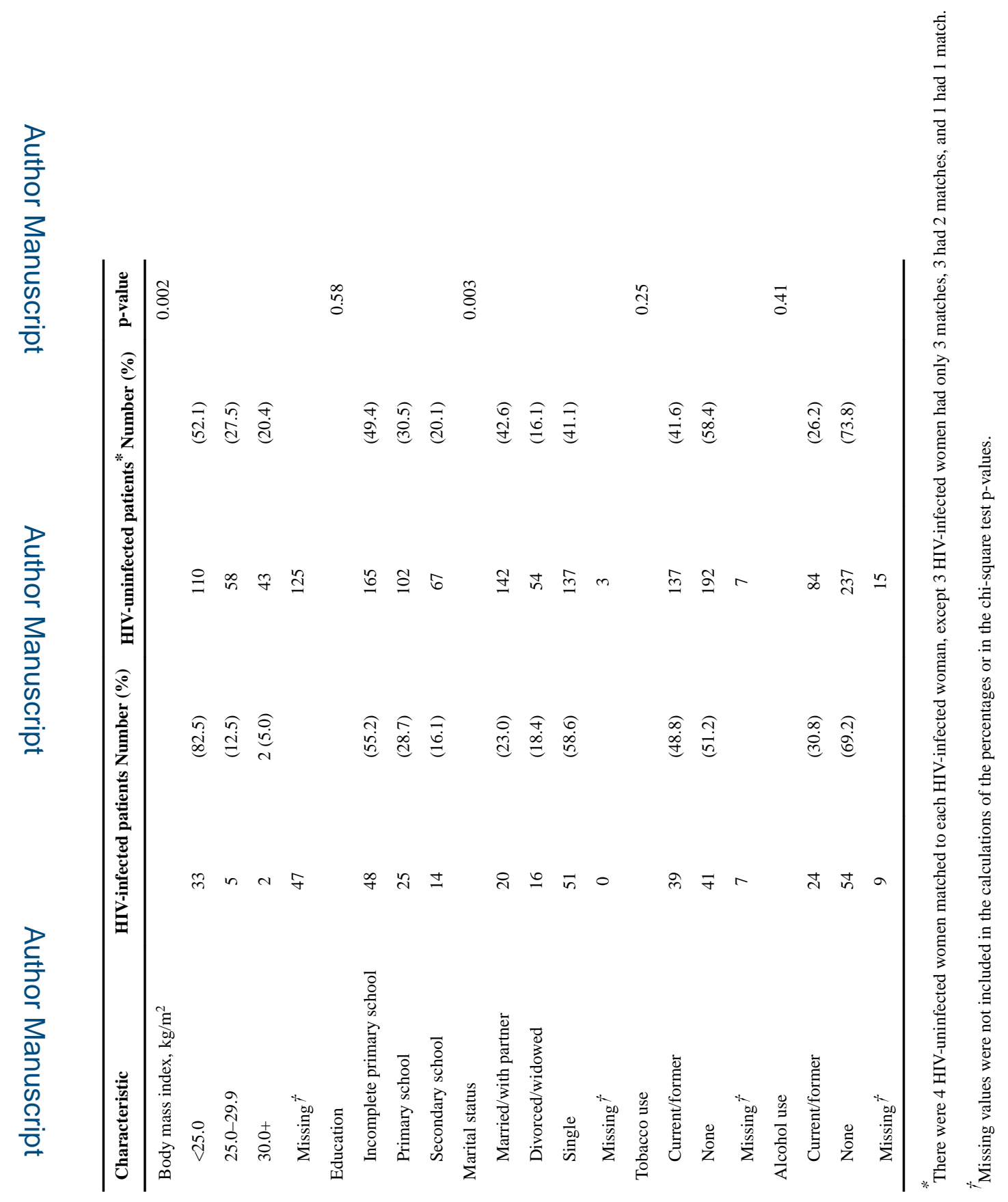

로을

AIDS. Author manuscript; available in PMC 2018 February 20. 


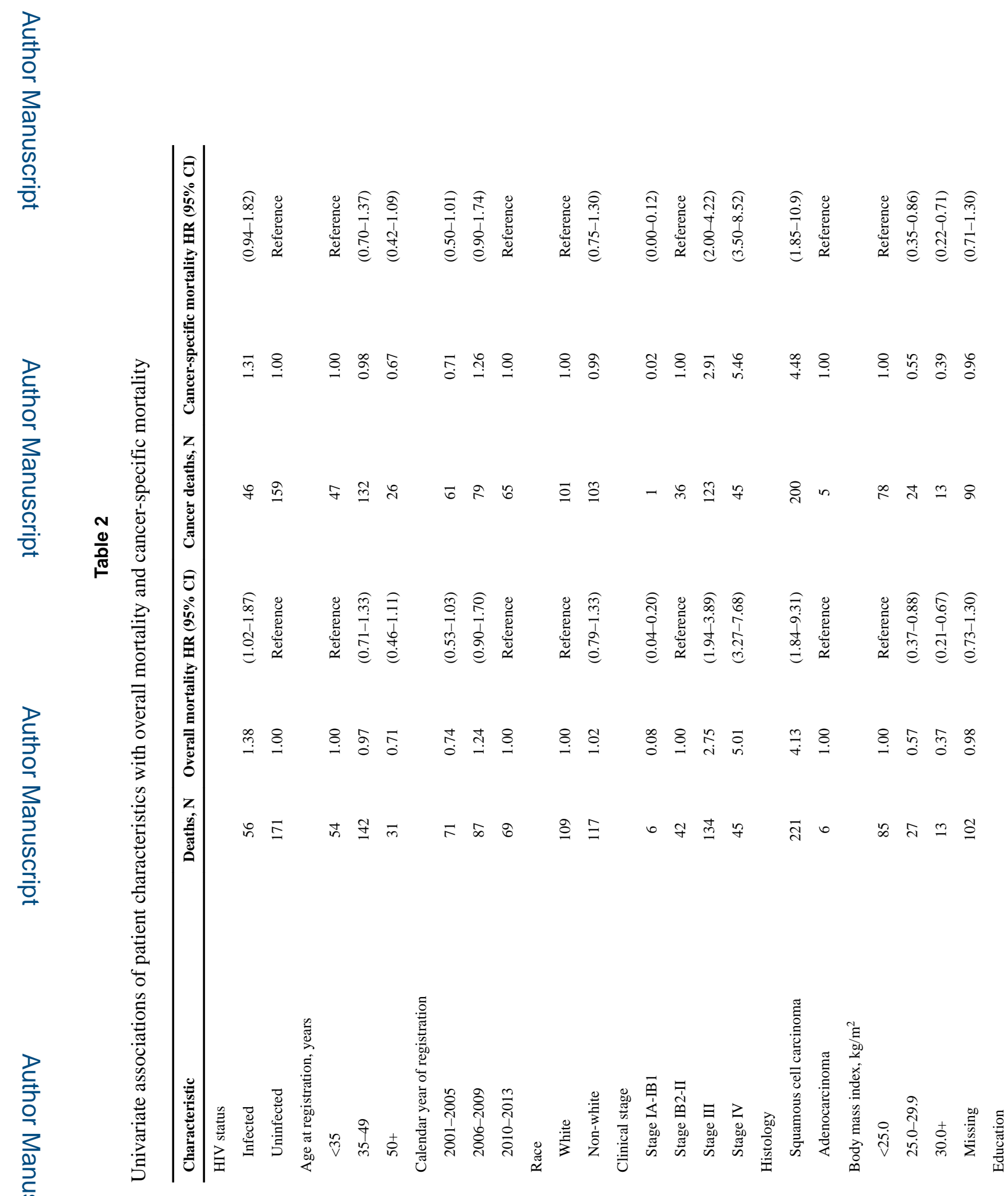

AIDS. Author manuscript; available in PMC 2018 February 20. 
Ferreira et al.

Page 15

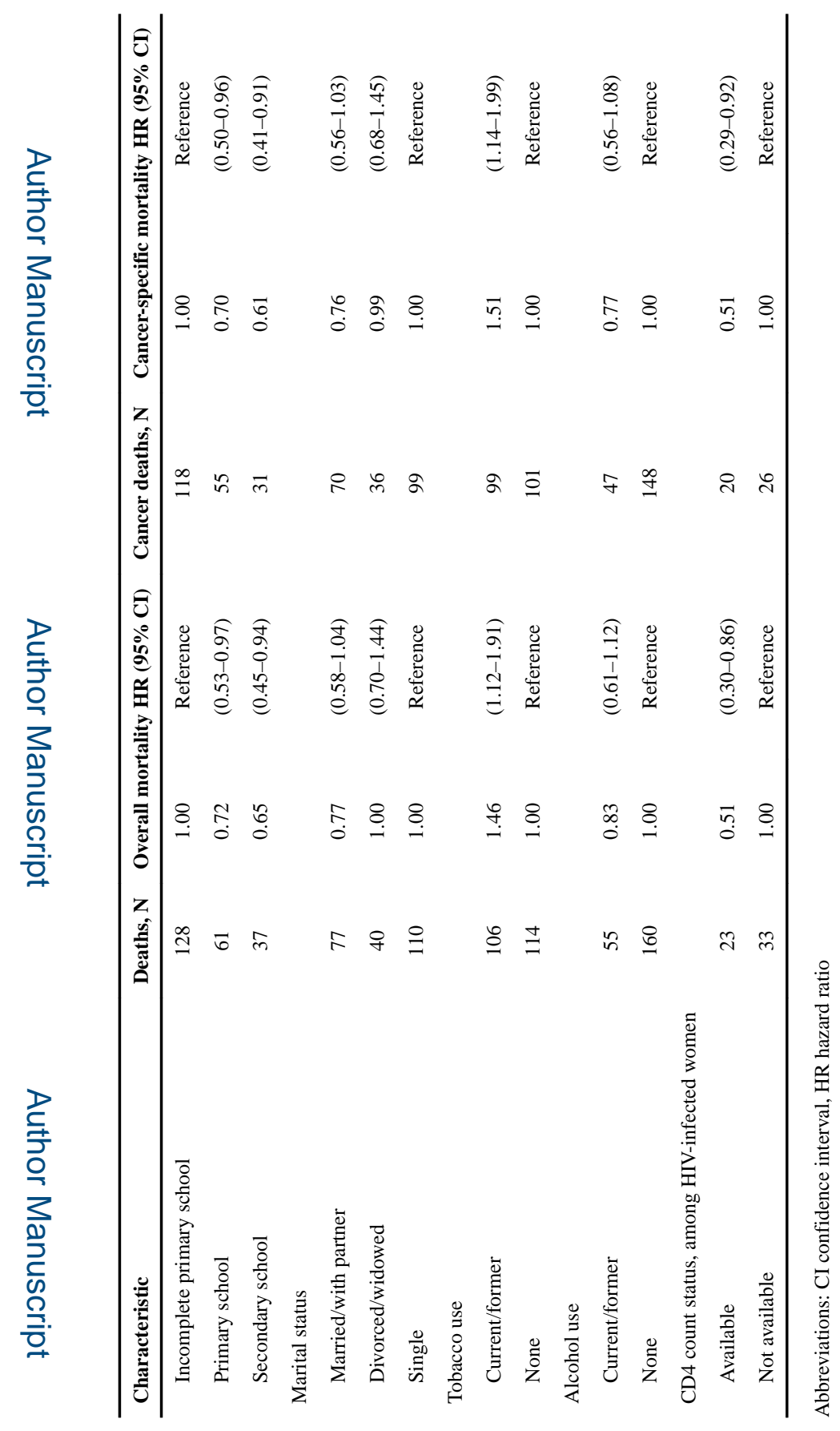

로을

AIDS. Author manuscript; available in PMC 2018 February 20. 


\section{Table 3}

Associations of HIV infection with overall mortality and cancer-specific mortality, overall and in patient subgroups

\begin{tabular}{lcc}
\hline Patient group & Overall mortality HR (95\% CI) & $\begin{array}{c}\text { Cancer-specific mortality HR } \\
(\mathbf{9 5 \%} \text { CI) }\end{array}$ \\
\hline All patients, unadjusted & $1.38(1.02-1.87)$ & $1.31(0.94-1.82)$ \\
All patients, adjusted for clinical stage & $1.29(0.95-1.75)$ & $1.18(0.85-1.65)$ \\
$\quad \begin{array}{l}\text { Models stratified by cancer treatment } \\
\quad \text { Patients treated with surgery, unadjusted }\end{array}$ & $8.70(1.59-47.5)$ & -- \\
$\quad \begin{array}{l}\text { Patients treated with radiation, adjusted for clinical stage and } \\
\text { brachytherapy }\end{array}$ & $1.22(0.82-1.82)$ & $0.96(0.62-1.48)$ \\
Models stratified by follow-up time, adjusted for clinical stage * & & \\
$\quad$ Early follow-up & & $0.99(0.69-1.42)$ \\
$\quad$ Late follow-up & $0.97(0.65-1.45)$ & $4.35(1.86-10.2)$ \\
\hline
\end{tabular}

Abbreviations: CI confidence interval, HR hazard ratio

Adjustment for clinical stage was accomplished using categories defined as stage IA/IB1, IB2/II, III, or IVA/IVB.

For overall mortality, follow-up time was divided at 1 year after cancer diagnosis. For cancer-specific mortality, follow-up time was divided at 2 years after cancer diagnosis. See Methods for details. 
Table 4

Associations of HIV infection with early outcomes of cancer treatment among patients treated with radiotherapy

\begin{tabular}{lcccc}
\hline & Complete response & Progression & Cancer-specific mortality & Other mortality \\
\hline \multicolumn{2}{l}{ HIV-infected patients } & & & \\
Events, N & 17 & 6 & 9 & 6 \\
HR $(95 \% \mathrm{CI})$ & $0.98(0.58-1.66)$ & $0.57(0.24-2.41)$ & $1.15(0.55-2.41)$ & Did not converge \\
HIV-uninfected patients & & & 1 \\
Events, N & 81 & 45 & 34 & 1.00 (reference) \\
HR $(95 \% \mathrm{CI})$ & 1.00 (reference) & 1.00 (reference) & 1.00 (reference) & \\
\hline
\end{tabular}

Abbreviations: CI confidence interval, HR hazard ratio

Results are from a Markov model (see Methods). 